redistribution of organ blood flow accounted for $<10 \%$ of the increase in arterial levels. Decrease in ammonia release in the renal vein may be sufficient to compensate for the increase in arterial ammonia due to shunted flow in Child Pugh A and B, but is insufficient in stage C.

Conclusion Portosystemic shunting may be centrally involved in the generation of hyperammonaemia in liver cirrhosis. This can be partially attenuated by increased urinary ammonia excretion. In patients with cirrhosis, decreased hepatic functions and metabolic changes may further increase the arterial ammonia levels.

Competing interests None declared.

\section{PTU-011 A NEW CONCEPT TO EXTEND RESECTABILITY OF LIVER TUMOURS: TWO STAGE SURGICAL STRATEGY USING AN IN-SITU-SPLIT PROCEDURE}

doi:10.1136/gutjnl-2012-302514c.11

${ }^{1,2} \mathrm{M}$ Donati, ${ }^{1,2} \mathrm{G}$ A Stavrou, ${ }^{1,2} \mathrm{~K}$ J Oldhafer. ${ }^{1}$ General and Visceral Surgery, Asklepios Barmbek Hospital, Hamburg, Germany; ${ }^{2}$ Asklepios Medical School, Hamburg, Germany

Introduction Today only $20 \%-25 \%$ of colorectal liver metastasis are resectable at initial presentation, despite the progress made in liver surgery over the last 25 years. ${ }^{1}$ The induction of liver hypertrophy by preoperative portal vein occlusion (Embolisation or Ligation) is the most used tool to prevent postoperative liver failure allows a Future Liver Remnant (FLR) growth of up to $20 \%-35 \%$ in 45 days. $^{2}$ For 1 year we have engaged a new method of achieving resectability in patients affected by extensive disease involving both lobes with insufficient future remnant liver volume (FRLV). ${ }^{3}$

Methods Between March and November 2011 six patients affected by liver tumours (4 colorectal liver metastasis (CRLM), 1 GISTmetastasis, 1 gallbladder carcinoma) were judged to be irresectable because of an insufficient RLV ( $<20 \%)$. Therefore all those patients were submitted to a two staged procedure: (1) Right portal vein ligation, in situ split procedure and additionally atypical resection of a metastasis in the FRL if needed. After CT controls with 3D reconstruction and volumetry (2) Extended right hemihepatectomy. ${ }^{3}$

Results Resectability was achieved in all patients around 2 weeks after step 1 (range 10-21 days). In five patients the FRL gained about $66 \%$ in volume (range $45 \%-95 \%$ ); the patients were operated and discharged without complications. One Patient (gallbladder carcinoma)-despite good volumetry (42\%)-suffered severe cholangitis postoperatively and died of consecutive liver failure 58 days after the second step operation.

Conclusion This method showed to be effective in patients initially judged to be irresectable. One possible explanation could be that the in-situ liver transection, causing disruption of intrahepatic portal collaterals, increases portal flow deprivation to the excluded segments and redistribution of hepatotrophic factors, accelerating future remnant liver growth. Patients with jaundice from biliary tract tumours seem not to be good candidates for this approach. The proposed new strategy has had value in extending resectability in patients suffering from extensive $\mathrm{CRLM},{ }^{3}$ reducing the risk of postoperatory liver failure, in our preliminary experience, more than other established methods.

Competing interests None declared.

\section{REFERENCES}

1. Capussotti L. Evolution of resectability criteria. In: Surgical treatment of colorectal liver metastases. Milan, Italy: Springer, 2011:27-34.

2. Hemming AW, Reed Al, Howard RJ, et al. Preoperative portal vein embolization for extended hepatectomy. Ann Surg 2003;237:686-91.

3. Oldhafer KJ, Donati M, Maghsoudi T, et al. Integration of 3D Volumetry, Portal Vein Transection and In Situ Split Procedure: A New Surgical Strategy for Inoperable Liver Metastasis. J Gastrointest Surg 2011.

\section{PTU-012 THE IMPACT OF COMORBIDITY ON POST LIVER TRANSPLANT SURVIVAL AND RESOURCE UTILISATION IN PATIENTS TRANSPLANTED FOR ACUTE LIVER FAILURE UTILISING THE CHARLSON COMORBIDITY INDEX}

doi:10.1136/gutjnl-2012-302514c.12

M A B Al-Freah,* B Kok, W Bernal, G Auzinger, N D Heaton, J G O'Grady, M A Heneghan, J A Wendon. Institute of Liver Studies, King's College Hospital, London, UK

Introduction The presence of comorbidities negatively impact post liver transplant (LT) survival for those transplanted with chronic liver disease.

Methods assess the impact of comorbidities on survival in patients transplanted for acute liver failure (ALF).

Results 176 patients underwent LT for ALF over 9 years. Median follow-up was 92 months (range 35-142). Median age was 33 years (17-67) and 122 (69.3\%) were females. Fifty-nine patients (33.5\%) were transplanted for Paracetamol induced ALF. Ninety-six (54.6\%) patients had $\geq 1$ comorbidity. The commonest comorbidity was renal dysfunction in 84 (48\%), pulmonary disease in $10(6 \%)$, connective tissue disease in $5(3 \%)$ and $2(1 \%)$ had diabetes. Patients with $\geq 1$ comorbidity had significantly increased 6 month (25\% vs $13 \%, p=0.046), 12$ month ( $27 \%$ vs $13 \%, p=0.023$ ) and overall mortality $(32 \%$ vs $17 \%, p=0.019)$. Similar results were demonstrated for graft survival. Recipient age $\geq 40$ years $(\mathrm{OR}=1.37,95 \%$ CI 1.02 to $1.86, \mathrm{p}=0.039)$, the presence of comorbidity $(\mathrm{OR}=1.46$, $95 \%$ CI 1.05 to $2.03, p=0.022)$ and renal dysfunction $(\mathrm{OR}=1.62$, $95 \%$ CI 1.18 to $2.23, \mathrm{p}=0.003$ ) were associated with increased post LT mortality on univariate analysis. However, only the presence of comorbidity ( $\mathrm{OR}=1.43,95 \% \mathrm{CI} 1.03$ to $1.98, \mathrm{p}=0.032)$ and renal dysfunction ( $\mathrm{OR}=1.59,95 \% \mathrm{CI} 1.15$ to $2.19, \mathrm{p}=0.004)$ were independently associated with mortality. Other recipient related, donor, or graft variables were not associated with mortality. Patients with $\geq 1$ comorbidity had significantly increased ICU length of stay (LoS) of 20 days ( 3 to 134) compared to those without comorbidities, 16 days $(2-102), p=0.005$.

Conclusion Pre- LT comorbidity as defined by the presence of $\geq 1$ comorbidity, significantly impairs overall post-LT patient and graft survival in patients transplanted for ALF. Patients with $\geq 1$ comorbidity had significantly increased ICU LoS which may suggest increased resource utilisation.

Competing interests None declared.

\section{REFERENCE}

1. Volk ML, et al. Modified Charlson comorbidity Index for predicting survival after liver transplantation. Liver Transplant 2007;13:1515-20.

\section{PTU-013 SINGLE USE OF ROMIPLOSTIM THROMBOPOIETIN ANALOGUE (TPO) IN SEVERE THROMBOCYTOPAENIA FOR OUTPATIENT PERCUTANEOUS LIVER BIOPSY IN PATIENTS WITH CHRONIC LIVER DISEASE (CLD): A RANDOMISED DOUBLE BLINDED PROSPECTIVE CLINICAL PILOT TRIAL}

doi:10.1136/gutjnl-2012-302514c.13

${ }^{1} \mathrm{P}$ Basu, ${ }^{*} \mathrm{~N}$ James Shah, ${ }^{2} \mathrm{~S}$ Farhat, ${ }^{2} \mathrm{~T}$ Nair. ${ }^{1}$ Gastroenterology and Liver Transplant, Columbia University, College of Physicians and Surgeons, New York, New York, USA ${ }^{2}$ Internal Medicine, North Shore University, Forest Hills, New York, USA

Introduction Thrombocytopaenia is a common entity in CLD with or without cirrhosis. Liver biopsy is the gold standard for diagnosis and prognosis of CLD. Platelet count is imperative before percutaneous liver biopsy. Platelet transfusion requires over night hospitalisation with transfusion associated morbidities and cost burden. 


\begin{tabular}{|c|c|c|c|c|c|c|c|}
\hline & $\begin{array}{l}\text { BL PLT } \\
\left(\times 10^{9} / l\right)\end{array}$ & $\begin{array}{l}\text { Pre0p } \\
\text { PLT }\left(\times 10^{9} / 1\right)\end{array}$ & $\begin{array}{l}4 \text { wk Post0p } \\
\left(\times 10^{9} / \mathrm{l}\right)\end{array}$ & $\begin{array}{l}\text { Mean Chg BL to } \\
\text { Pre0p }\left(\times 10^{9} / l\right)\end{array}$ & $\begin{array}{l}\text { Mean Chg Pre0p to } \\
4 \text { wk Post0p }\left(\times 10^{9} / \mathrm{l}\right)\end{array}$ & $\begin{array}{l}\text { Mean Chg BL to } \\
4 \text { wk Post0p }\left(\times 10^{9} / l\right)\end{array}$ & Cost (WAC) \\
\hline Romiprostim & $58.7^{*}$ & $232 \dagger$ & $366.2 \ddagger$ & $173.3 \dagger$ & $192.9 \ddagger$ & $307.5 \ddagger$ & $\$ 2284$ \\
\hline
\end{tabular}

${ }^{*} \mathrm{p}=\mathrm{ND}$ vs PLT.

$t \mathrm{p}<0.05$ vs PLT TRF and Elthrombopag.

$\neq p<0.001$ vs PLT TRF and Elthrombopag.

$\S p<0.001$ vs PLT TRF.

Romiplostim, a fusion protein TPO, is a hormone that regulates platelet production approved in idiopathic thrombocytopenic purpura (ITP). This study evaluates single use of Romiplostim 2 week prior to liver biopsy to avoid biopsy related morbidity and mortality.

Methods 65 patients $(\mathrm{n}=65)$, (mean age: 56 years; M:F-2:1) with Hepatitis C: $37 / 65$ (57\%); hepatitis B (HBV) 7 (15.5\%), Alcoholic Cirrhosis 10 (15\%); Non-Alcoholic steato-hepatits (NASH) 3 (5\%), Primary biliary cirrhosis (PBC) $6(9 \%)$ with pre-biopsy mean platelet count $77 \mathrm{k}$; Mean MELD score 20, mode fibrotic score F4 were randomised in blinded fashion into three groups: Group A $(n=18)$, received seven units of platelet transfusion at night for the morning procedure. Group B ( $\mathrm{n}=23$ ) received Romiplostim $500 \mu \mathrm{g}$ sc given 2 weeks prior to the procedure, and Group $C(n=24)$ Elthrombopag orally $75 \mathrm{mg}$ /day for 2 weeks. PLT-CT was repeated $2 \mathrm{~h}$ prior and Post-biopsy in 4 weeks in all groups. Inclusion criteria: CLD with thrombocytopaenia.

\section{Results}

Conclusion This pilot study demonstrates that single use of Romiplostim is efficacious, cost-effective, and safe without side effects for liver biopsy with severe thrombocytopaenia. Single use of Romiplostim should be considered before Trans jugular intra-hepatic porto-systemic shunts or portal haemodynamic procedures and prior to surgical interventions with severe thrombocytopaenia. A large randomised clinical trial is needed for further validation.

Competing interests None declared.

\section{PTU-014 LEVELS OF CARE AND OUTCOMES IN DECOMPENSATED CIRRHOSIS: A DESCRIPTIVE STUDY}

doi:10.1136/gutjnl-2012-302514c.14

${ }^{1} \mathrm{P}$ Berry, ${ }^{*}{ }^{2} \mathrm{~S}$ Thomson, ${ }^{1} \mathrm{~A}$ Ahmed, ${ }^{3} \mathrm{M}$ Davies, ${ }^{1} \mathrm{~A}$ Ala. ${ }^{1}$ Centre for Gastroenterology, Hepatology and Nutrition, Frimley Park Hospital NHS Foundation Trust, Frimley, UK; ${ }^{2}$ Gastroenterology and Hepatology, Western Sussex Hospitals NHS Trust, Worthing, UK; ${ }^{3}$ Intensive Care Unit, Frimley Park Hospital NHS Foundation Trust, Frimley, UK

Introduction As hospital admissions due to decompensated cirrhosis increase it is important to characterise the severity of disease and establish "best practice" in secondary care units. While many studies have reported on patients in the ITU setting, we have expanded this evaluation to explore patient outcome according to level of inpatient care provided; ward (level 0-1), medical HDU (2) or ICU (3).

Methods Prospective analysis of consecutive decompensated cirrhosis patients admitted to the liver service of a district general hospital between November 2010 and November 2011).

Results 66 patients accounted for 153 separate presentations. ALD accounted for 54 patients (78\%). Of those with ALD 24 (44\%) were a first presentation, $22(41 \%)$ continued to drink against advice and eight were abstinent. Alcoholic hepatitis ( $\mathrm{AH}$ ) was diagnosed in 24, with median discriminant function (DF) of 49. 16 (23\%) were admitted to ICU (multiple organ failure 12, variceal bleeding without organ failure 4). $88 \%$ were mechanically ventilated, $81 \%$ received inotropic support and 56\% haemofiltration. 14 (20\%) were admitted to medical HDU and the $36(61 \%)$ remained on the ward. Median MELD and UKELD scores were not significantly different between groups; ITU 16 and 57, HDU 20 and 58, Ward 13 and 55 respectively. Overall median length of stay was 11.5 days. LOS was highest in HDU patients ( $23 \mathrm{~d}$ vs ward $11 \mathrm{~d}$ and ITU $17 \mathrm{~d}, \mathrm{p}=0.04$ ). In-hospital mortality was $5 \%$ in ward patients, $14 \%$ in HDU patients and $36 \%$ in ITU patients $(p=0.01)$, with 90 -day mortality rising to $8 \%, 21 \%$ and $50 \%$ respectively. Six patients with extrahepatic organ dysfunction received a "ceiling of care" decision, whereby active treatment on HDU was offered but organ support on ICU was not; these patients did not differ from ICU patients in terms of age, active drinking, liver failure scores or SOFA score. The natural history of these patients was characterised by rising MELD despite optimum therapy and late development of extra-hepatic organ dysfunction (median period from admission $17 \mathrm{~d}$ vs $1 \mathrm{~d}$, $\mathrm{p}=\mathrm{NS})$. All six died.

Conclusion This cohort of cirrhotic patients displayed evidence of advanced liver disease. Median UKELD in ward patients was above the limit required for consideration of liver transplant and median DF indicated a high risk of death in those diagnosed with $\mathrm{AH}$. Prolonged hospital stays and ward based convalescence were required, especially in those escalated to HDU, but mortality was lower than that commonly perceived for this patient cohort. Patients who are not offered advanced organ support are identified after gradual deterioration despite optimal ward care. We propose the concept of a "medical HDU" model where hepatologists are able to escalate levels of care locally before the onset of organ dysfunction.

Competing interests None declared.

\section{PTU-015 EXPANDED CD14HI/CD16 + MONOCYTES IN ACUTE LIVER INJURY (ALI): ANGELS OR DEMONS?}

doi:10.1136/gutjnl-2012-302514c.15

${ }^{1} \mathrm{R}$ D Abeles, ${ }^{*}{ }^{1,2} \mathrm{C}$ G Antoniades, ${ }^{1} \mathrm{G}$ K Manakkat Vijay, ${ }^{2} \mathrm{~N}$ Vergis, ${ }^{1} \mathrm{~J}$ R Maggs, ${ }^{1} \mathrm{~W}$ Bernal, ${ }^{1} \mathrm{D}$ L Shawcross, ${ }^{1} \mathrm{~J}$ A Wendon, ${ }^{1} \mathrm{D}$ Vergani. ${ }^{1}$ Institute of Liver Studies, King's College London at King's College Hospital, London, UK; ${ }^{2}$ Imperial College London, London, UK

Introduction CD14hi/CD16+ monocytes resemble tissue macrophages and normally constitute $\sim 5 \%$ of circulating monocytes. Expansion of CD14hi/CD16+ monocytes has been implicated in the perpetuation of liver injury and hepatic fibrosis in chronic liver disease. We therefore wanted to explore whether CD14hi/CD16+ monocytes are implicated in ALI; show features of activated endothelial adhesion (CD11b) and chemotaxis (CCR5); and phenotypically resemble classically activated "M1" or alternatively activated "M2" macrophages. "M1" macrophages secrete pro-inflammatory cytokines, express high levels of HLA-DR and CD86 and are implicated in tissue damage. "M2" macrophages, are immunoregulatory, express scavenger receptors such as CD163 and are involved in tissue repair.

Methods Blood was sampled from patients within $24-48 \mathrm{~h}$ of admission consisting of 33 hyper-Acute Liver Failure (hyperALF: 